\title{
Research on Express Smart Locker Location Considering Customer Satisfaction under
}

\section{E-commerce Environment}

\author{
Ao Huang \\ College of Management \\ Wuhan University of Science \& Technology \\ Wuhan, China \\ E-mail: 277701862@qq.com
}

\author{
Tao Chen \\ College of Management \\ Wuhan University of Science \& Technology \\ Wuhan, China
}

\begin{abstract}
Express smart locker location optimization scheme is proposed based on the delivery demand under E-commerce environment, to improve delivery network efficiency and to solve customer satisfaction problem. This paper takes into account the influence of distance on consumers' pickup service satisfaction through constructing customer satisfaction function, and then establishes multi-objective mathematical model with the minimum economic cost and maximum customer satisfaction. The model is transformed to single-object model through linear weighted sum. Finally, the model is applied in a computational example to get a feasible solution, and the model effectiveness is verified. The result indicates that the model can effectively settle the problem of express smart locker location.
\end{abstract}

Keywords-E-commerce environment; express smart locker; location; customer satisfaction

\section{INTRODUCTION}

In recent years, the rapid development of e-commerce has driven swift growth of express delivery demand. The sharp increase in express delivery quantity has resulted in heavy pressure on goods processing and delivery for express delivery enterprises. Thus, the "last-mile" issue at the endpoint of express delivery becomes more prominent. As a result, a series of problems such as low delivery efficiency, poor service quality and low customer satisfaction restrict sound development of e-commerce ${ }^{[1]}$. Express smart locker mode as an emerging express delivery mode has become an effective way to solve the "last-mile" issue of express delivery and contributes to resolving such problems as high delivery cost, goods safety, inconvenient pickup and inconsistency between delivery time and consumers' pickup time ${ }^{[2]}$. Express smart locker mode is an important means of logistics service innovation in endpoint delivery, and endpoint delivery mode based on smart locker is an inexorable trend. However, it is generally believed that numerous problems exist in express smart locker layout planning ${ }^{[3]}$. Therefore, this paper aims to explore and solve the problems of express smart locker layout planning, in the hope of providing decision reference for practical promotion of the locker.

Many scholars have studied self-service parcel pickup mode. Wang Dongliang and Yu Zhenning(2016) analyzed endpoint delivery development situation of Zhuhai and the problems existing in express smart locker development by combining the specific conditions of Zhuhai city development, and put forward the suggestions on express smart locker development ${ }^{[4]}$. Wu Min and Xu Junjie(2016) proposed critical development motivations of convenient pickup point - cost, service, profit and environment according to the environment of Chinese e-commerce logistics industry, and discussed the optimal development mode of three types of developers ${ }^{[5]}$. Shi Shubiao and Huang Youfang et al.(2015) came up with automatic logistic terminal equipment in campus express and studied the implementation feasibility of automatic logistic terminal equipment in campus express through qualitative analysis and quantitative analysis ${ }^{[6]}$. Punakivi(2001) proposed two main modes of unattended delivery - unattended reception box and delivery box, and found unattended reception box more cost-saving through comparing them from the perspective of cost ${ }^{[7]}$. Mckinnon and Tallam(2013) classified major types of unattended delivery, assessed the safety respectively, confirmed the safety problem of unattended delivery and proposed the countermeasures ${ }^{[8]}$. $\mathrm{Xu}(2008)$ probed into consumers' perception for current delivery service and the perception of consumers and online retailers for unattended delivery, and found British consumers were not optimistic about unattended delivery and that the opinions of consumers and online retailers on future delivery differed ${ }^{[9]}$. Present researches mostly focus on development status of express smart locker mode, promotion of the mode and development countermeasures, while there are few researches about express smart locker location.

Hu Zhijie and Guo Wenqian(2012) discovered that customers mainly took into account of cost, distance and time during selecting express smart locker mode and that distance factor was the most important factor influencing customer satisfaction [10]. Zhang Zongxiang and Yang Chao(2012) investigated the influence of facility coverage range on user satisfaction and established facility location model based on gradually coverage ${ }^{[11]}$. Yang Pengjue and $\mathrm{Hu} \mathrm{Hao}(2014)$ considered that terminal point should be as close to customers as possible within the service range ${ }^{[12]}$. Thus, this paper takes into account the influence of distance on consumers' pickup 
service satisfaction and studies express smart locker location through constructing customer satisfaction function.

\section{CUSTOMER SATISFACTION FUNCTION}

Customers often have an anticipant distance range between their position and the express smart locker. Within the distance range, customers will feel entirely satisfied, or else their satisfaction will decline gradually ${ }^{[13]}$. When the distance exceeds the maximum distance that a customer can bear, the customer will feel completely dissatisfied and refuse to pick up the goods by himself. Hence, this paper introduces fuzzy membership function to reflect customer satisfaction.

In this paper, it is assumed that customer satisfaction for express smart locker is a non-increasing function of distance from customers' position to the locker and the function obeys concave-convex function curve, as shown in Fig.1. Every customer has an optimal distance $\left[0, s_{1}\right]$. Within the distance, there is no penalty cost and the customer satisfaction is the highest. Meanwhile, there is no penalty cost within the distance $\left(s_{1}, s_{2}\right)$, but customer satisfaction is affected. When the distance exceeds $s_{2}$, the customer will feel completely dissatisfied and refuse to accept self-service pickup. Thus, the delivery center needs to deliver the goods again. In this case, penalty cost will occur. On this basis, the satisfaction of customer i can be expressed as fuzzy membership function $U\left(s_{i}\right)$ of distance $\left(s_{i}\right)$ from the customer's position to the express smart locker. The formula is as follows:

$$
U\left(s_{i}\right)=\left\{\begin{array}{cc}
1 & s_{i} \in\left[0, s_{1}\right] \\
1-\left(\frac{s_{i}-s_{1}}{s_{2}-s_{1}}\right)^{k_{i}} & s_{i} \in\left(s_{1}, s_{2}\right) \\
0 & s_{i} \in\left[s_{2}, \infty\right)
\end{array}\right.
$$

Wherein, $\boldsymbol{k}_{\boldsymbol{i}}$ is distance sensitivity coefficient .Within the interval $\left[s_{1}, s_{2}\right]$, the curve is concave when $k_{i}<1$; the curve is convex when $k_{i}>1$; the function is a straight line when $k_{i}=1$.

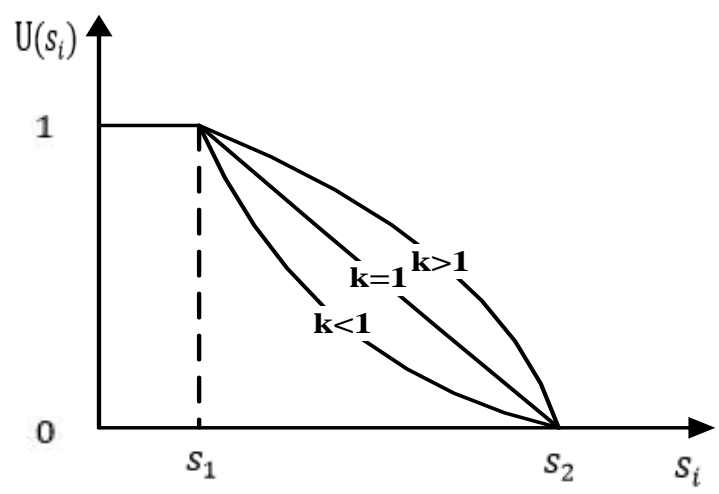

\section{PICKUP POINT LOCATION OPTIMIZATION MODEL}

\section{A. Problem description and hypothesis}

For the problem of express smart locker layout location, this paper takes single delivery center - multiple alternative locker points - multiple demand points as the objects of study. The location optimization problem can be defined as follows: the position of delivery center, the position of alternative point and the quantity demanded are known; the vehicles dispatched by the delivery center serve for each express smart locker, and each locker can meet customer demand; the total cost is minimum and customer satisfaction is maximum through rational selection of pickup point. The following hypotheses are made for the sake of modeling:

(1) The operation and management cost for each alternative point is same, and only fixed cost, delivery cost and penalty cost are considered;

(2) Customer satisfaction is a distance-related function, and the penalty cost will be incurred when the maximum distance that a customer can bear exceeds;

(3) Each demand point and the quantity demanded are known, and each demand point has only one express smart locker;

(4) The delivery center is known, and the goods are transported to the express smart locker from the delivery center;

(5) The freight rate of delivery vehicles remains unchanged, and the delivery cost is related to the distance;

(6) The fixed cost of diverse alternative points is different.

\section{B. Modeling}

It is assumed that $M$ express smart lockers are needed; there are $\mathrm{m}$ alternative points and $\mathrm{n}$ demand points. $z_{i}=1$ means the locker is built at point $\mathrm{i} ; z_{i}=0$ means the locker is not built at the alternative point $\mathrm{i} ; x_{i j}=1$ means the alternative point $\mathrm{i}$ provides the service for the demand point $\mathrm{j} ; x_{i j}=0$ means the alternative point $i$ does not provide the service for the demand point $\mathrm{j} ; s_{i j}$ represents the distance between the alternative point $\mathrm{i}$ and the demand point $\mathrm{j} ; \boldsymbol{q}_{j}$ represents the quantity demanded by the demand point $\mathrm{j} ; d_{i}$ represents the transportation distance between the delivery center to the alternative point $\mathrm{i}$; $a_{i j}=1$ means the distance between the alternative point $\mathrm{i}$ and the demand point $\mathrm{j}$ exceeds the maximum distance that the customer can accept; $a_{i j}=0$ means the distance between the alternative point $i$ and the demand point $j$ does not exceed the maximum distance that the customer can accept; The construction cost of alternative locker point $i$ is $A_{i}$; the freight per unit distance is $\mathrm{B}$; the penalty cost per unit distance is $\mathrm{C}$.

Fig.1 Schematic diagram of customer’s distance satisfaction 
Objective functions:

$$
\begin{gathered}
\max F_{1}=\frac{\sum_{i=1}^{m} \sum_{j=1}^{n} U\left(s_{i j}\right) x_{i j} q_{j}}{\sum_{j=1}^{n} q_{j}} \\
\min F_{2}=\sum_{i=1}^{m} A_{i} z_{i}+\sum_{i=1}^{m} B d_{i} z_{i}+\sum_{i=1}^{m} \sum_{j=1}^{n} s_{i j} x_{i j} q_{j}
\end{gathered}
$$

Constraint conditions:

$$
\begin{gathered}
\sum_{i=1}^{m} x_{i j}=1 \quad i=1, \ldots, m \quad j=1, \ldots, n \\
x_{i j} \leq z_{i} \quad i=1, \ldots, m \quad j=1, \ldots, n \\
\sum_{i=1}^{m} z_{i}=M \quad i=1, \ldots, m \\
\sum_{j=1}^{n} x_{i j} q_{j} \leq Q \quad i=1, \ldots, m \quad j=1, \ldots, n \\
x_{i j}=\left\{\begin{array}{ll}
1 & s_{i j} \leq S_{-} \\
0 & s_{i j}>S_{-}
\end{array} \quad i=1, \ldots, m \quad j=1, \ldots, n\right. \\
0,1\} \quad \begin{array}{ll}
a_{i j}=\{0,1\} \quad z_{i}=\{0,1\} & i=1, \ldots, m \quad j=1, \ldots, n
\end{array}
\end{gathered}
$$

Objective functions (2) and (3) mean the location model owns maximum customer satisfaction and minimum total cost is the objective.(4) and (5) show each demand point has only one locker service, and lockers have the point responsible for delivery;(6) means the number of express smart lockers built is same with the planned number;(7) means the capacity of locker is larger than the parcel quantity delivered;(8) indicates that the penalty cost will be incurred when the distance between the customer to the locker exceeds the maximum distance that the customer can accept.

\section{MOdEL SOlution METHOD}

In this paper, the locker point location is a multi-objective planning model. The solution methods of multi-objective planning model mainly include linear weighted sum, index weighted product, ideal point, stratified sequence and main objective etc. Since the importance degree of customer satisfaction objective function and total cost objective function is different, linear weighted sum method is applied to solve the model. First of all, customer satisfaction maximization is transformed to the minimization:

$$
\min F_{1}=1-\frac{\sum_{i=1}^{m} \sum_{j=1}^{n} U\left(s_{i j}\right) x_{i j} q_{j}}{\sum_{j=1}^{n} q_{j}}
$$

Because the dimension of multi-objective function is not unified, the range method is adopted for dimensionless treatment of objective function ${ }^{[14]}$. The objective function after dimensionless treatment is as follows:

$$
\begin{gathered}
F_{1}^{*}=\left(F_{1}-\min F_{1}\right) /\left(\max F_{1}-\min F_{1}\right) \\
F_{2}^{*}=\left(F_{2}-\min F_{2}\right) /\left(\max F_{2}-\min F_{2}\right)
\end{gathered}
$$

The weight vector is $W=\left(w_{1}, w_{2}\right)$, and $w_{1}+w_{2}=1$, $w_{1}, w_{2} \in[0,1]$.Then, $F=w_{1} F_{1}^{*}+w_{2} F_{2}^{*}$.In accordance with field survey results, the weight vector can be gained roughly, $W=(0.45,0.55)$. Thus, the generalized objective function is obtained as follows:

$$
\min F=0.45 F_{1}^{*}+0.55 F_{2}^{*}
$$

Through linear weighting, the multi-objective optimization problem is transformed to single objective optimization problem as shown in Formula (13). Finally, LINGO programming is applied for solving the formula. LINGO belongs to a modeling language of optimization problem and contains many common functions which can be used. In addition, it is convenient for inputting, solving and analyzing large-scale optimization problem ${ }^{[15]}$.

\section{EXAMPLE ANALYSIS}

In order to improve regional delivery efficiency, an express enterprise decided to build express smart lockers in a region. There are 18 demand points and 1 delivery center in the region. The enterprise drafted 6 alternative locker points. Daily quantity demanded by each demand point is shown in Table 1 , and the distance between each alternative point to the delivery center is shown in Table 2. It is assumed that the customer's satisfactory distance for the locker point is within $200 \mathrm{~m}$; the maximum distance that the customer can bear is $800 \mathrm{~m}$; the distance sensitivity coefficient is 1 . The fixed cost of each alternative point is shown in the table. The freight per unit distance is $0.2 \mathrm{Yuan} / \mathrm{m}$, and the penalty cost per unit distance is $0.004 \mathrm{Yuan} / \mathrm{m}$. Due to the budget constraint, the final number of express smart lockers is 3 at most.

DAILY QUANTITY DEMANDED BY EACH DEMAND POINT

\begin{tabular}{cccccccccc}
\hline Demand Point & 1 & 2 & 3 & 4 & 5 & 6 & 7 & 8 & 9 \\
\hline Quantity Demanded & 10 & 4 & 8 & 5 & 7 & 4 & 8 & 11 & 7 \\
\hline \hline Demand Point & 10 & 11 & 12 & 13 & 14 & 15 & 16 & 17 & 18 \\
\hline Quantity Demanded & 5 & 9 & 4 & 3 & 4 & 45 & 49 & 52 & 70 \\
\hline
\end{tabular}


PRESS

DISTANCE BETWEEN EACH ALTERNATIVE POINT TO EACH DEMAND POINT AND THE DELIVERY CENTER

\begin{tabular}{|c|c|c|c|c|c|c|}
\hline $\begin{array}{l}\text { Alternative } \\
\text { Point } \\
\text { Point }\end{array}$ & 1 & 2 & 3 & 4 & 5 & 6 \\
\hline 1 & 0 & 1443 & 200 & 343 & 103 & 216 \\
\hline 2 & 278 & 1216 & 393 & 616 & 1345 & 869 \\
\hline 3 & 442 & 1100 & 326 & 579 & 174 & 1189 \\
\hline 4 & 678 & 1729 & 533 & 0 & 348 & 556 \\
\hline 5 & 777 & 2069 & 780 & 138 & 627 & 1497 \\
\hline 6 & 41 & 890 & 140 & 284 & 218 & 839 \\
\hline 7 & 208 & 1859 & 478 & 130 & 325 & 396 \\
\hline 8 & 103 & 1345 & 442 & 775 & 0 & 888 \\
\hline 9 & 477 & 923 & 846 & 1341 & 935 & 1136 \\
\hline 10 & 1443 & 0 & 1314 & 2121 & 1578 & 1693 \\
\hline 11 & 1119 & 108 & 806 & 1485 & 1056 & 1226 \\
\hline 12 & 763 & 1713 & 705 & 212 & 693 & 869 \\
\hline 13 & 1214 & 793 & 882 & 865 & 1356 & 1016 \\
\hline 14 & 110 & 1297 & 55 & 225 & 317 & 214 \\
\hline 15 & 433 & 755 & 193 & 739 & 631 & 553 \\
\hline 16 & 2275 & 860 & 2009 & 2656 & 2281 & 2515 \\
\hline 17 & 513 & 2114 & 688 & 454 & 679 & 1115 \\
\hline 18 & 285 & 1396 & 734 & 925 & 121 & 327 \\
\hline Delivery Center & 1230 & 350 & 709 & 1069 & 892 & 1550 \\
\hline
\end{tabular}

TABLE II. FIXED COST OF EACH ALTERNATIVE POINT

\begin{tabular}{ccccccc}
\hline Alternative Point & 1 & 2 & 3 & 4 & 5 & 6 \\
\hline Fixed Cost & 130 & 110 & 150 & 100 & 80 & 200
\end{tabular}

TABLE IV MODEL SOLUTION RESULtS

\begin{tabular}{cccccccccc}
\hline Demand Point & 1 & 2 & 3 & 4 & 5 & 6 & 7 & 8 & 9 \\
\hline Alternative Point & 1 & 1 & 5 & 5 & 5 & 1 & 1 & 5 & 1 \\
\hline \hline Demand Point & 10 & 11 & 12 & 13 & 14 & 15 & 16 & 17 & 18 \\
\hline Alternative Point & 2 & 2 & 1 & 2 & 1 & 1 & 2 & 1 & 5 \\
\hline
\end{tabular}

The results computed by LINGO are shown in Table IV. The optimal solutions are alternative points $\{1,2,5\}$. In other words, the express smart lockers are built at the three alternative points 1,2 and 5 . The alternative point 1 serves for demand points $\{1,2,6,7,9,12,14,15,17\}$. The alternative point 2 serves for demand points $\{10,11,13,16\}$. The alternative point 5 serves for demand points $\{3,4,5,8,18\}$. In this way, the economic cost is the least and customer satisfaction is the largest. So, the location is reasonable and satisfies the requirements of location model. The model is feasible and practical.

\section{CONCLUSION}

Express smart locker layout has an important effect on endpoint delivery and locker use ratio. The quantity of locker points decides the expenditure of fixed cost, while the point distribution decides basic structure of delivery endpoint network and concerns vehicle transport route and distance ${ }^{[16]}$. Different point layout decides pickup efficiency which influences consumers' acceptance of such delivery mode. Thus, it is required to reasonably distribute the lockers in a bid to adapt to the delivery demand under e-commerce environment, enhance delivery network efficiency and customer satisfaction, and reduce total cost ${ }^{[17]}$.

This paper researches the issue of express smart locker location, introduces customer satisfaction function and establishes a double-objective model with maximum customer satisfaction and minimum economic cost. Meanwhile, linear weighted sum is employed to transform multi-objective optimization problem to single objective model, and the model effectiveness is verified through a computational example. Therefore, the decision maker can resolve the problems existing in the process of express smart locker location according to the model.

\section{REFERENCES}

[1] Yang Juping, Yang Changchun; Yao Xuanxia. Research on the "LastMile" Issue in the E-Commerce Logistics System [J]. Journal of Business Economics, 2014 (4): 16-22. (In Chinese)

[2] Wang Jiaqi, Zou En. Analysis of Application of Smart Express Parcel Cabinets in Online Shopping Endpoint Logistics [J]. Logistics Technology, 2015, 34(5):58-60. (In Chinese)

[3] Xu Junjie, Jiang Ling, Li Yiliang. Consumer's Selecting Willingness of Picking up Parcels: An Empirical Retest for Service Driving Hypothesis [J]. Chinese Journal of Management, 2014, 11(12):1850-1857. (In Chinese)

[4] Wang Dongliang, Yu Zhenning. Countermeasures for Developing Smart Delivery Cabinet System against Intelligent City Background: In the Case of Zhuhai [J]. Logistics Technology, 2016, 35(5). (In Chinese)

[5] Wu Min, Xu Junjie, Development Motivation of Convenient Pickup Point_-Based on Domestic Industrial Environment of E-commerce Logistics [J]. Logistics Technology, 2016, 39(2):51-53. (In Chinese)

[6] Shi Shubiao, Huang Youfang, Yan Wei, Research on Automatic Logistic Terminal Equipment Application in Campus Express [J]. Computer Simulation, 2015, 32(9): 421-424. (In Chinese)

[7] Punakivi M. Solving the last mile issue: reception box or delivery box? [J]. International Journal of Physical Distribution \& Logistics Management, 2001, 31(6):427-439.

[8] Mckinnon A C, Tallam D. Unattended delivery to the home: an assessment of the security implications [J]. International Journal of Retail \& Distribution Management, 2013, 31(1):30-41.

[9] Xu M. The last mile of e-commerce - unattended delivery from the consumers and eTailers' perspectives [J]. International Journal of Electronic Marketing \& Retailing, 2008, 2(1).

[10] Hu Zhijie, Guo Wenqian, Wu Hao. A Feasibility Study on the Distribution Mode of Intelligent Self- extracting Cabinet with Customer Satisfaction [J]. Logistics Engineering and Management, 2012, 17(5):35-39. (In Chinese)

[11] Zhang Zongxiang, Yang Chao, Chen Zhongwu. Stochastic Gradual Covering Location Models and Approach Based on Service Quality [J]. Industrial Engineering and Management, 2012, 17(5):35-39. (In Chinese)

[12] Yang Pengjue, $\mathrm{Hu}$ Hao, Wang Junjia et al. Location Model for Terminals of Urban Distribution under E-commerce Environment [J]. Industrial Engineering and Management, 2014, 19(1):35-40. (In Chinese)

[13] Li Zilin, Kong Weiwei. Evaluation of Transport System for Transfer Passenger at Traffic Hub in Tianjin Binhai New Area [J]. Journal of Tianjin Institute of Urban Construction, 2009, 15(1):28-32. (In Chinese)

[14] Zhao Rui, Hu Xiong, He Hongdi. E-commerce logistics distribution routing optimization considering customer satisfaction [J]. Journal of Shanghai Maritime University, 2015(3):64-70. (In Chinese)

[15] Yuan Xinsheng, Application of LINGO and Excel in Mathematical Modeling [M]. Science Press, 2016. (In Chinese) 
[16] Chen Yiyou, Chen Yiheng. Research on pickup point location model and algorithm in presence of gradual coverage [J]. Application Research of Computers, 2016, 33(8). (In Chinese)

[17] Zhou Lin, Lin Yun, Wang Xu et al. Integrated optimization for multiclass terminal location-heterogeneous vehicle routing of urban distribution under online shopping [J]. Computer Integrated Manufacturing Systems, 2016, 22(4):1139-1147. (In Chinese) 